\section{Padrões alimentares de crianças de 13 a 35 meses de idade e associação com características maternas}

\section{Eating patterns among children aged 13 to 35 months and association with maternal characteristics}

\section{Estándares alimentarios en niños de 13 a 35 meses de edad y su asociación con características maternas}

Eduarda Gomes Bogea 1

Maylla Luanna Barbosa Martins 1

Wyllyane Rayana Chaves Carvalho 1

Soraia Pinheiro Machado Arruda 2

Ana Karina Teixeira da Cunha França 1

Antonio Augusto Moura da Silva 1

doi: 10.1590/0102-311X00072618

\section{Correspondência}

E. G. Bogea

Universidade Federal do Maranhão.

O objetivo foi identificar os padrões alimentares em crianças e verificar a sua associação com características maternas. Foram estudadas 1.185 crianças de 13 a 35 meses de idade. O consumo alimentar foi investigado por meio do recordatório alimentar de 24 horas e os padrões alimentares identificados pela análise fatorial por componentes principais, seguida de rotação ortogonal varimax. Utilizou-se a modelagem hierarquizada por meio da regressão de Poisson com estimativa robusta da variância para calcular as razões de prevalências. Quatro padrões alimentares foram identificados: comum brasileiro, lácteo/mingau, saudável e não saudável. A variância explicada por esses fatores foi de 34,3\%, sendo que o padrão comum brasileiro foi o que mais contribuiu com a variância proporcional. Destaca-se o padrão lácteo/mingau, em que o leite materno apresentou carga fatorial negativa, apontando para a sua substituição pelos leites de vaca e modificados. Os dados revelam que a caracterização dos padrões alimentares das crianças diverge segundo as características maternas, como a idade, escolaridade e número de filhos. Três dos quatro padrões encontrados são representados por alimentos ricos em amido, gordura saturada e açúcar simples, e pobres em vitaminas, minerais e fibras. Multiparidade, menor escolaridade materna e idade materna menor que 20 anos foram associadas ao menor consumo de alimentos considerados saudáveis e importantes para o desenvolvimento infantil.

Nutrição da Criança; Consumo Alimentar; Mães; Análise Fatorial
R. Barão de Itapari 155, São Luís, MA 65020-070, Brasil. eduardabogea@gmail.com

1 Universidade Federal do Maranhão, São Luís, Brasil. 2 Universidade Estadual do Ceará, Fortaleza, Brasil. 


\section{Introdução}

Dentre os vários aspectos relacionados com a criança, a nutrição representa um requisito fundamental para o seu crescimento e desenvolvimento, principalmente nos primeiros anos de vida 1. Essa fase merece atenção especial, pois é nela que os riscos relacionados com os agravos nutricionais são bem evidenciados 2 e são construídos os alicerces dos hábitos alimentares 3 .

O conhecimento do consumo alimentar de crianças e a compreensão dos diversos fatores que o influenciam podem proporcionar um maior entendimento da complexa relação entre dieta e saúde na infância 3,4. Geralmente, os estudos epidemiológicos que avaliam dieta são baseados em cálculos da ingestão energética total, dos macros e micronutrientes ou na análise de um nutriente isolado 5,6. Entretanto, esse tipo de análise não leva em consideração a complexidade da dieta humana e as combinações de nutrientes, o que limita tal análise 7 .

Diante disso, as análises de identificação de padrões alimentares emergiram como abordagem alternativa e complementar para avaliar a dieta, pois expressam melhor a dieta consumida por uma dada população 7,8. Um dos métodos mais usados para a definição de padrões alimentares utiliza a correlação entre os vários alimentos para descrever o padrão alimentar geral, por meio de análise fatorial com o método de extração dos componentes principais 3,9 .

Estudos têm mostrado que os padrões alimentares dos indivíduos podem ser influenciados por fatores biológicos, nutricionais e socioeconômicos 7,10. Em crianças menores de dois anos, além desses fatores, o comportamento e as características da mãe merecem destaque, pois esta é a principal referência de cuidados à criança. A adequação desses cuidados depende diretamente da habilidade e do desempenho da mãe e de sua escolaridade, disponibilidade de tempo, renda, estilo de vida e do seu estado de saúde física e mental 4,11,12.

Um estudo de coorte realizado no Japão, que acompanhou crianças de 16-24 meses, definiu dois padrões alimentares: "frutas, hortaliças e alimentos proteicos" e "guloseimas e bebidas açucaradas". Na análise de associação, variáveis como escolaridade materna e número de irmãos mais velhos foram associadas aos hábitos alimentares de crianças 13 .

Em um trabalho realizado no Sul do Brasil 3, com crianças de 12 a 48 meses, foram extraídos cinco padrões alimentares: "leites", "base", "adulto", "bebidas" e "lanches". Foram encontradas diferenças entre os grupos para quase todas as variáveis socioeconômicas estudadas, como o nível econômico e a escolaridade da mãe. Um estudo realizado em Minas Gerais, com pré-escolares, encontrou que filhos de famílias com renda superior a 1/2 salário mínimo apresentaram chance mais de duas vezes maior de consumo do padrão "não saudável" 14.

A maior parte dos trabalhos brasileiros que utilizou padrões alimentares investigou a população adulta, sendo escassos os estudos realizados com o público infantil, principalmente na Região Nordeste. Dessa forma, o presente trabalho teve como objetivo identificar os padrões alimentares de crianças no segundo ano de vida e investigar sua associação com as características maternas em uma capital do Nordeste.

\section{Métodos}

\section{Delineamento do estudo}

Trata-se de um estudo transversal realizado com base em um trabalho prospectivo de coorte intitulado Etiologia do Nascimento Pré-termo e Consequências dos Fatores Perinatais para a Saúde Infantil: Coortes de Nascimento de Duas Cidades Brasileiras, São Luis (MA) e Ribeirão Preto (SP) - BRISA. Este estudo utilizou dados da coorte na cidade de São Luís, Maranhão, coletados em dois períodos: no nascimento, de janeiro a dezembro de 2010, e no segundo ano de vida, de abril de 2011 a janeiro de 2013.

\section{População e amostra em estudo}

Em 2010, ocorreram 21.401 nascimentos hospitalares nos dez hospitais de São Luís, nos quais foi realizada a pesquisa, o que correspondeu a $94,7 \%$ do total dos nascimentos do município. Desses, 
7.133 ( 1 a cada 3 ) foram sistematicamente selecionados, dois quais 5.475 eram partos de residentes elegíveis para entrevista. Assim, a corte incluiu no nascimento 5.236 crianças, sendo que não puderam ser entrevistadas 239 mães por recusa ou alta precoce. Desse total, 70 eram natimortos, restando 5.166 casos para análise. Os métodos detalhados utilizados na coorte de nascimento foram descritos em outra publicação 15.

Para o seguimento foi selecionada uma subamostra de 2.135 crianças da coorte de nascimentos BRISA, composta por todos os nascidos de baixo peso e/ou pré-termos e/ou gemelares (853), e uma vez e meia este número de crianças a termo (1.282), selecionadas por amostragem aleatória simples, que constituíram o grupo controle. Dessas, 893 não foram localizadas ou não compareceram para a avaliação realizada de 13-35 meses de idade, sendo então avaliadas 1.242 crianças. Foram excluídos 57 casos de alimentação atípica, mediante a resposta negativa das mães à pergunta: "Ontem, a criança foi alimentada como de costume?”, independentemente do motivo. Dessa forma, a amostra final do estudo foi constituída por 1.185 crianças, resultando em $44,5 \%$ de perdas.

\section{Procedimentos de coleta de dados}

Os dados socioeconômicos e demográficos foram coletados por meio de um questionário padronizado aplicado no seguimento do 20 ano de vida. As informações referentes ao consumo alimentar foram obtidas por inquérito alimentar recordatório de 24 horas (R24h). Todas as entrevistas foram realizadas com as mães e/ou responsáveis das crianças, como recomendado pela literatura ${ }^{16}$. Para garantir a padronização da coleta de dados, a equipe de pesquisadores e entrevistadores foi devidamente treinada para a utilização de questionários e aplicação do inquérito alimentar.

A aplicação do R24h foi realizada no domicílio e, inicialmente, a mãe foi questionada se no dia anterior a criança havia se alimentado como sempre. Independentemente da resposta, foi solicitado que a mãe descrevesse detalhadamente os alimentos, o modo de preparo e quantificasse os itens consumidos, em medidas caseiras. Um álbum fotográfico foi usado para facilitar a recordação dos itens ingeridos pela criança no dia anterior.

Antes da digitação dos dados do consumo alimentar, em programa específico, foi checada a qualidade das informações coletadas e realizada a quantificação dos alimentos e bebidas de forma padronizada, com o auxílio da Tabela para Avaliação de Consumo Alimentar em Medidas Caseiras 17. Preparações regionais ou que não constavam no banco de dados do programa foram incluídas de acordo com os ingredientes e as quantidades descritas no R24h. Posteriormente, os dados dos R24h foram convertidos em energia e nutrientes usando-se o Programa Virtual Nutri Plus (http://virtualnutriplus.com.br/ Portal/Default.aspx), versão 2010, da Universidade de São Paulo. Após essa etapa, houve a exportação e organização dos dados para uma planilha eletrônica do programa Excel (https://products.office. com/), versão 2010.

Em função da dificuldade de mensuração do volume diário de leite materno consumido por aquelas crianças que ainda eram amamentadas, utilizou-se a metodologia de Drewett et al. 18 por ser de fácil aplicação e não exigir tecnologia. Por esse método, estima-se o volume de leite materno consumido pela quantidade (em quilocalorias) da alimentação complementar e pela idade da criança em dias. Tais variáveis compõem um modelo de regressão linear múltipla proposto por Drewett et al. 18, em que $Y$ é a estimativa do consumo de leite materno, $X$ ' é a idade (dias) e X" é o consumo de alimentos complementares em quilocalorias: $\mathrm{Y}=755,0-0,48 \mathrm{X}$ - $0,59 \mathrm{X}$ ”. Segundo os mesmos autores, o número de mamadas por dia e o consumo de outros alimentos são variáveis que expressam melhor o consumo de leite materno do que apenas a duração das mamadas.

Para este trabalho, a variável resposta foi o padrão alimentar das crianças, e as variáveis independentes foram raça e sexo da criança e variáveis que representavam as características maternas: idade ( $<20$ anos; 20 a 34; $\geq 35$ anos); escolaridade ( 1 a 4 anos; 5 a $8 ; 9$ a $11 ; 12$ ou mais anos); recebimento de benefício do Bolsa Família (sim; não); situação conjugal da mãe (casada; união consensual; sem companheiro); número de moradores da casa ( $\leq 4$ pessoas; $>4$ pessoas); classificação econômica brasileira (A/B; C; D/E), de acordo com a Associação Brasileira de Empresas de Pesquisa (ABEP); exercício de atividade remunerada (sim; não); e número de filhos que moram com a mãe (1 filho; 2 a 4; mais de 5 filhos). 


\section{Tratamento das variáveis dietéticas}

Para se obter a variável dietética usada na análise de derivação dos padrões alimentares, as quantidades de alimentos consumidas foram convertidas de medidas caseiras para consumo diário de cada alimento em gramas $(\mathrm{g})$ ou mililitros $(\mathrm{mL})$, de acordo com a Pesquisa de Orçamentos Familiares 2008-2009 19 .

Quando o alimento não foi consumido pelo indivíduo, atribuiu-se o valor 0. Os 134 alimentos identificados no R24h foram reunidos em 22 grupos, considerando-se a semelhança da sua composição nutricional. Foram mantidos separados os itens alimentares citados por pelo menos $80 \%$ dos sujeitos, e foram excluídos aqueles grupos alimentares citados por menos de $3 \%$ dos entrevistados.

\section{Análise estatística}

Como a amostra deste estudo foi composta por todas as crianças nascidas de baixo peso e/ou pré-termo e/ou gemelares e uma vez e meia este número de controles, as probabilidades de seleção foram diferentes para estes grupos. Devido à amostragem complexa e ao percentual de 44,5\% de perdas de elegíveis (950 de 2.135), as estimativas foram ponderadas pelo inverso da probabilidade de seleção e levou-se também em conta a ausência de resposta.

Para a definição dos padrões alimentares foi utilizado o método de análise fatorial por componentes principais (ACP), seguido de rotação ortogonal do tipo varimax, para se identificar os padrões alimentares do grupo estudado. A adequação dos dados à análise fatorial foi confirmada por meio do coeficiente de Kaiser-Meyer-Olkin (KMO). O número de fatores retidos foi definido com base nos seguintes critérios: componentes com autovalores maiores que 1,5, gráfico de Cattel e o significado conceitual dos padrões identificados. Cada componente principal foi interpretado baseando-se nos alimentos com cargas fatoriais $\geq 0,3$ ou $\leq-0,3$, que é considerada uma importante contribuição para o padrão ${ }^{7}$. Dentro de um componente, cargas negativas indicam associação inversa do item alimentar e cargas positivas indicam associação direta.

Os componentes principais foram rotulados com base na composição nutricional dos alimentos de cada fator. Cada criança recebeu um escore para cada fator retido. Os padrões alimentares foram categorizados em quartis. O quartil superior da distribuição representou maior aderência ao padrão.

Foi feita uma modelagem hierarquizada usando-se a regressão de Poisson com estimativa robusta de variância, para estimar as razões de prevalência (RP) das variáveis independentes (características maternas) com as variáveis dependentes (padrões alimentares). A constituição dos blocos e sua ordenação obedeceram a um modelo teórico prévio de determinação e precedência temporal. Variáveis demográficas da criança (raça e sexo), que não são explicadas por nenhuma variável antecedente, foram colocadas no primeiro bloco. Em seguida, foram consideradas as variáveis socioeconômicas da mãe (escolaridade e classificação econômica brasileira), que determinam as variáveis reprodutivas que, por sua vez, determinam as variáveis demográficas, reprodutivas e estado nutricional da mãe (situação conjugal, idade da mãe no parto, número de filhos, número de pessoas na casa e estado nutricional). Variáveis do recém-nascido e a variável "outra gravidez após o nascimento da criança avaliada” constituíram o terceiro bloco. No quarto bloco foi incluída a variável de assistência social (recebimento do Bolsa Família). A ordenação do terceiro e quarto blocos foi temporal.

As variáveis que apresentaram $\mathrm{p}<0,05$, em qualquer nível do modelo teórico, foram consideradas significantes e passaram a compor o grupo das variáveis do nível seguinte. Esse procedimento foi repetido até o nível final. A interpretação da RP foi feita no nível que a variável pertence. As estimativas foram calculadas por pontos e por intervalos de 95\% de confiança (IC95\%). O nível de significância adotado foi de $5 \%$. As análises estatísticas foram realizadas no programa Stata (https://www.stata. com), versão 12.0 .

Esta pesquisa foi submetida ao Comitê de Ética em Pesquisa (CEP) do Hospital Universitário da Universidade Federal de Maranhão (UFMA) e aprovada sob o parecer no 223/09 e registro 350/08, em conformidade com os requisitos fundamentais da Resolução no 196/96 e suas complementares do Conselho Nacional de Saúde. 


\section{Resultados}

Das 1.185 crianças estudadas, 51,3\% eram do sexo masculino e 74,4\% tinham cor da pele não branca. Ao se analisar as características socioeconômicas das mães, observou-se que $71,7 \%$ tinham entre 20 e 34 anos, 59,7\% viviam em união consensual e 69,4\% não recebiam o benefício do Bolsa Família. A maioria das mães (62,8\%) tinha de 9 a 11 anos de estudos, 19,3\% de 5 a 8, 15,2\% tinham 12 ou mais anos de estudos e a minoria (2,7\%) de 1 a 4 anos. Em relação à ABEP, 55,4\% estavam inseridas na classe D/E. Mais de $2 / 3(69,1 \%)$ das mães moravam com menos de quatro pessoas em casa e $49 \%$ tinham apenas um filho. A prevalência de agravos nutricionais nas mães foi de 48,8\%, sendo 39,9\% de excesso de peso e $8,9 \%$ de desnutrição.

O valor do teste para a avaliação das correlações entre os itens alimentares e da adequação do uso da análise fatorial para a identificação dos padrõea alimentares foi satisfatório e apropriado para ACP $(\mathrm{KMO}=0,609)$. Considerando-se o scree plot, com base no qual foram identificados 4 pontos no maior declive, e autovalores $\geq 1,5$, foi determinada a extração de quatro fatores. A extração desses componentes explicou 34,3\% da variância total após a rotação dos fatores (Tabela 1).

Os padrões alimentares foram definidos da seguinte forma: comum brasileiro, lácteo/mingau, saudável e não saudável. O padrão comum brasileiro explicou a maior proporção da variância total (9,3\%), representando melhor o consumo alimentar da amostra avaliada (Tabela 2).

Após o ajuste, em análise hierarquizada, notou-se que quanto menor a escolaridade materna, maior foi a aderência a esse padrão ( 1 a 4 anos: RP = 2,71; IC95\%: 1,47-4,98; 5 a 8 anos: RP = 2,21; IC95\%: 1,38-3,53; 9 a 11 anos: RP = 1,68; IC95\%: 1,10-2,56). Filhos de mães com idades iguais ou superiores a 35 anos apresentaram menor aderência ao padrão comum brasileiro $(\mathrm{RP}=0,30$; IC95\%: 0,15-0,58) e os de mães com idades inferiores a 20 anos, maior aderência ( $R P=1,40$; IC95\%: 1,081,80). Percebeu-se, ainda sobre o padrão comum brasileiro, que quanto maior o número de filhos, maior foi a aderência a este padrão (1 filho: $\mathrm{RP}=0,61$; IC95\%: 0,48-0,77; mais de 5 filhos: $\mathrm{RP}=1,52$; IC95\%: 1,05-2,21) (Tabela 1).

O padrão lácteo/mingau não apresentou associação com as variáveis investigadas (Tabela 3). Em relação ao padrão saudável, a análise bivariada apontou menor aderência para as crianças de mães com menor escolaridade e das classes econômicas D e E. Após análise hierarquizada, apenas duas categorias da variável escolaridade permaneceram com significância estatística: 1 a 4 anos $(\mathrm{RP}=0,17$; IC95\%: 0,04-0,80) e 9 a 11 anos (RP = 0,67; IC95\%: 0,51-0,88) (Tabela 4).

O padrão não saudável apresentou associação com as variáveis idade da mãe e número de filhos. Notou-se menor aderência a esse padrão para os filhos únicos (RP = 0,80; IC95\%: 0,64-1,00) e para os filhos de mães com idades superiores a 35 anos ( $R P=0,40$; IC95\%: 0,23-0,70) (Tabela 5).

\section{Discussão}

Quatro padrões alimentares foram identificados entre as 1.185 crianças estudadas: o comum brasileiro, lácteo/mingau, saudável e o não saudável. A variância explicada por esses fatores foi de 34,3\%, sendo que o padrão comum brasileiro foi o que mais contribuiu com a variância proporcional. A caracterização dos padrões alimentares das crianças divergiu segundo as características maternas, como idade, escolaridade e número de filhos.

Como pontos fortes do trabalho, destacamos ser este estudo de base populacional, no qual foi realizada amostragem probabilística de 1/3 dos nascimentos ocorridos em São Luís em 2010. Além disso, a amostragem do seguimento teve tamanho adequado para distinguir diferenças na aderência dos padrões entre as categorias das variáveis analisadas.

Como limitação, registramos a utilização de um único R24h para construir os padrões alimentares das crianças investigadas, visto que um único recordatório não permite minimizar as questões relacionadas à variabilidade intraindividual do consumo de alimentos. Embora esse método não represente a ingestão habitual de um indivíduo, a sua precisão aumenta quando a amostra é constituída por número elevado de indivíduos, como foi observado nesta investigação. Além disso, o R24h, como todo método de mensuração do consumo alimentar, apresenta limitações e vieses próprios, como o viés de 
Tabela 1

Razões de prevalência (RP) brutas e ajustadas e intervalos de 95\% de confiança (IC95\%) para a associação entre características maternas e o padrão alimentar comum brasileiro em crianças da coorte de nascimento BRISA. São Luís, Maranhão, Brasil, 2010-2013.

\begin{tabular}{|c|c|c|c|c|}
\hline & RP bruta (IC95\%) & Valor de $\mathrm{p}$ & RP ajustada (IC95\%) & Valor de $p$ \\
\hline \multicolumn{5}{|l|}{ Nível 1: variáveis da criança } \\
\hline Sexo & & 0,431 & & 0,456 \\
\hline Feminino & Ref. & & Ref. & \\
\hline Masculino & $1,09(0,88-1,34)$ & & $1,08(0,88-1,33)$ & \\
\hline Raça & & 0,059 & & 0,060 \\
\hline Não branca & Ref. & & Ref. & \\
\hline Branca & $0,78(0,60-1,01)$ & & $0,78(0,60-1,01)$ & \\
\hline \multicolumn{5}{|c|}{ Nível 2: variáveis socioeconômicas da mãe } \\
\hline Escolaridade (anos) & & $<0,001$ & & 0,002 * \\
\hline 1 a 4 & $2,69(1,53-4,72)$ & & $2,71(1,47-4,98)$ & \\
\hline 5 a 8 & $2,08(1,38-3,14)$ & & $2,21(1,38-3,52)$ & \\
\hline 9 a 11 & $1,59(1,08-2,33)$ & & $1,68(1,10-2,56)$ & \\
\hline 12 ou mais & Ref. & & Ref. & \\
\hline Classificação econômica brasileira & & 0,112 & & 0,386 \\
\hline $\mathrm{A} / \mathrm{B}$ & Ref. & & Ref. & \\
\hline $\mathrm{C}$ & $1,08(0,79-1,47)$ & & $0,79(0,56-1,11)$ & \\
\hline $\mathrm{D} / \mathrm{E}$ & $1,28(0,91-1,80)$ & & $0,83(0,57-1,21)$ & \\
\hline \multicolumn{5}{|c|}{$\begin{array}{l}\text { Nível 3: variáveis demográficas, reprodutivas e estado } \\
\text { nutricional da mãe }\end{array}$} \\
\hline Situação conjugal & & 0,063 & & 0,823 \\
\hline Casada & Ref. & & Ref. & \\
\hline União consensual & $1,41(1,05-1,90)$ & & $1,08(0,80-1,48)$ & \\
\hline Sem companheiro & $1,42(1,00-2,02)$ & & $1,12(0,78-1,61)$ & \\
\hline Idade da mãe no parto (anos) & & $<0,001$ * & & $<0,001$ * \\
\hline$<20$ & $1,21(0,96-1,53)$ & & $1,40(1,08-1,80)$ & \\
\hline 20 a 34 & Ref. & & Ref. & \\
\hline$\geq 35$ & $0,33(0,11-0,63)$ & & $0,30(0,15-0,58)$ & \\
\hline Número de filhos & & $<0,001 *$ & & 0,000 * \\
\hline 1 & $0,72(0,58-0,89)$ & & $0,61(0,48-0,77)$ & \\
\hline 2 a 4 & Ref. & & Ref. & \\
\hline$\geq 5$ & $1,62(1,08-2,44)$ & & $1,52(1,05-2,21)$ & \\
\hline Quantas pessoas moram na casa & & 0,047 * & & 0,445 \\
\hline$\leq 4$ & Ref. & & Ref. & \\
\hline$>4$ & $1,24(1,00-1,53)$ & & $1,09(0,88-1,34)$ & \\
\hline Estado nutricional da mãe & & 0,791 & & 0,910 \\
\hline Eutrofia & Ref. & & Ref. & \\
\hline Desnutrição & $1,05(0,73-1,51)$ & & $1,03(0,72-1,47)$ & \\
\hline Excesso de peso & $0,93(0,75-1,17)$ & & $0,96(0,77-1,19)$ & \\
\hline \multicolumn{5}{|l|}{ Nível 4: variável de assistência social } \\
\hline Recebe Bolsa Família & & $<0,001$ * & & 0,145 \\
\hline Não & Ref. & & Ref. & \\
\hline Sim & $1,43(1,16-1,76)$ & & $1,17(0,94-1,46)$ & \\
\hline
\end{tabular}

Ref.: referência.

Notas: foram avaliadas 1.185 crianças de 13 a 35 meses de idade. Nível 1: feito ajuste para as variáveis deste nível; Nível 2: feito ajuste para as variáveis deste nível e para as variáveis significantes $(p<0,05)$ do nível anterior (Nível 1); Nível 3: feito ajuste para as variáveis deste nível e para as variáveis significantes $(p<0,05)$ dos níveis anteriores (Níveis 1 e 2); Nível 4: feito ajuste para as variáveis deste nível e para as variáveis significantes ( $<$ <,05) dos níveis anteriores (Níveis 1, 2 e 3).

* Valor de $p<0,05$. 
Tabela 2

Cargas fatoriais dos padrões alimentares identificados em 1.185 crianças de 13 a 35 meses de idade, da coorte de nascimento BRISA (1ํ acompanhamento). São Luís, Maranhão, Brasil, 2010-2013.

\begin{tabular}{|c|c|c|c|c|}
\hline \multirow[t]{2}{*}{ Grupos alimentares } & \multicolumn{4}{|c|}{ Padrões alimentares } \\
\hline & Comum brasileiro & Lácteo/Mingau & Saudável & Não saudável \\
\hline Pães & 0,639 & & & \\
\hline Café & 0,512 & & & \\
\hline Gorduras & 0,336 & & & \\
\hline Leguminosas & 0,459 & & & $-0,444$ \\
\hline Farináceos & & 0,632 & & \\
\hline Produtos açucarados & & 0,558 & & \\
\hline Leite de vaca e modificados & & 0,659 & & \\
\hline Leite materno & & $-0,500$ & & \\
\hline Hortaliças e folhosos & $-0,513$ & & 0,311 & \\
\hline Tubérculos & $-0,546$ & & 0,350 & \\
\hline Carnes e víceras & & & 0,574 & \\
\hline Arroz e macarrão & & & 0,590 & \\
\hline Frutas e sucos de fruta/Polpa & & & 0,338 & \\
\hline Sopa industrializada/Macarrão instantâneo & & & $-0,465$ & \\
\hline Refrigerantes/Sucos artificiais & & & & 0,654 \\
\hline Biscoitos/Bolos simples & & & & 0,568 \\
\hline Lanches não saudáveis & & & & 0,267 \\
\hline Número de itens & 6 & 4 & 6 & 3 \\
\hline Variância proporcional (\%) & 9,27 & 8,87 & 8,56 & 7,57 \\
\hline Variância acumulada (\%) & 9,27 & 18,14 & 26,70 & 34,27 \\
\hline Coeficente KMO & 0,609 & & & \\
\hline
\end{tabular}

KMO: coeficiente de Kaiser-Meyer-Olkin.

aferição. Neste estudo, algumas estratégias foram utilizadas para a redução do viés de aferição, como o treinamento dos entrevistadores e a padronização das medidas dos alimentos.

Ademais, 44,5\% dos selecionados para o seguimento não compareceram para entrevista. Para amenizar essa limitação, as estimativas de associação foram ponderadas também levando-se em conta a ausência de resposta. Está bem demonstrado que ponderar pelo inverso da probabilidade de seleção é preferível ao uso das estimativas não ponderadas quando há diferenças na composição da amostra do seguimento em comparação à amostra original, e que este procedimento é capaz de corrigir para viés de seleção. Entretanto, a sua performance depende de um modelo correto para predizer a participação na fase de seguimento do estudo ${ }^{20}$. Dessa forma, mesmo que a ponderação pelo inverso da probabilidade de seleção seja capaz de remover viés, é possível que o viés de seleção residual ainda esteja presente. A ponderação não foi realizada no processo da ACP, pois o programa utilizado, Stata 12.0, não permite a incorporação de pesos amostrais, sendo esta uma limitação do estudo.

A não mensuração do consumo de leite materno no R24h também é uma limitação. Para a estimação do consumo do leite foi usada a equação de Drewett et al. ${ }^{18}$. Esse método, ainda que seja validado, foi desenvolvido em outra população, que pode apresentar características diferentes de consumo do leite materno. Apesar disso, esse método é de baixo custo, fácil aplicabilidade e leva em consideração a quantidade calórica consumida e a idade da criança para a sua quantificação. Uma outra limitação é que o método da ACP não escapa de críticas quanto à subjetividade que imprime na determinação do número de fatores a serem extraídos na agregação dos grupos.

Os quatro padrões extraídos explicaram 34,3\% da variância total após a rotação dos fatores. Tal resultado foi similar ao encontrado no trabalho de Matos et al. 21 com crianças de 18 a 23 meses de 
Tabela 3

Razões de prevalência (RP) brutas e ajustadas e intervalos de 95\% de confiança (IC95\%) para a associação entre características maternas e o padrão alimentar lácteo/mingau em crianças da coorte de nascimento BRISA. São Luís, Maranhão, Brasil, 2010-2013.

\begin{tabular}{|c|c|c|c|c|}
\hline & RP bruta (IC95\%) & Valor de $p$ & RP ajustada (IC95\%) & Valor de $p$ \\
\hline \multicolumn{5}{|l|}{ Nível 1: variáveis da criança } \\
\hline Sexo & & 0,693 & & 0,684 \\
\hline Feminino & Ref. & & Ref. & \\
\hline Masculino & $1,04(0,84-1,29)$ & & $1,04(0,85-1,29)$ & \\
\hline Raça & & 0,860 & & 0,858 \\
\hline Não branca & Ref. & & Ref. & \\
\hline Branca & $1,02(0,80-1,30)$ & & $1,02(0,80-1,30)$ & \\
\hline \multicolumn{5}{|c|}{ Nível 2: variáveis socioeconômicas da mãe } \\
\hline Escolaridade (anos) & & 0,544 & & 0,465 \\
\hline 1 a 4 & $0,56(0,25-1,26)$ & & $0,52(0,22-1,22)$ & \\
\hline 5 a 8 & $0,88(0,62-1,25)$ & & $0,81(0,54-1,21)$ & \\
\hline 9 a 11 & $0,93(0,70-1,23)$ & & $0,87(0,63-1,20)$ & \\
\hline 12 ou mais & Ref. & & Ref. & \\
\hline Classificação econômica brasileira & & 0,869 & & 0,787 \\
\hline $\mathrm{A} / \mathrm{B}$ & Ref. & & Ref. & \\
\hline C & $1,05(0,78-1,41)$ & & $1,14(0,82-1,58)$ & \\
\hline $\mathrm{D} / \mathrm{E}$ & $1,02(0,73-1,44)$ & & $1,18(0,56-1,77)$ & \\
\hline \multicolumn{5}{|c|}{$\begin{array}{l}\text { Nível 3: variáveis demográficas, reprodutivas e estado } \\
\text { nutricional da mãe }\end{array}$} \\
\hline Situação conjugal & & 0,812 & & 0,885 \\
\hline Casada & Ref. & & Ref. & \\
\hline União consensual & $0,99(0,76-1,30)$ & & $1,01(0,76-1,33)$ & \\
\hline Sem companheiro & $1,08(0,78-1,50)$ & & $1,08(0,76-1,53)$ & \\
\hline Idade da mãe no parto (anos) & & 0,509 & & 0,476 \\
\hline$<20$ & $1,10(0,85-1,44)$ & & $1,06(0,80-1,42)$ & \\
\hline 20 a 34 & Ref. & & Ref. & \\
\hline$\geq 35$ & $1,19(0,84-1,69)$ & & $1,24(0,87-1,76)$ & \\
\hline Número de filhos & & 0,562 & & 0,455 \\
\hline 1 & $1,10(0,89-1,36)$ & & $1,12(0,89-1,41)$ & \\
\hline 2 a 4 & Ref. & & Ref. & \\
\hline$\geq 5$ & $0,84(0,42-1,65)$ & & $0,79(0,40-1,56)$ & \\
\hline Quantas pessoas moram na casa & & 0,691 & & 0,646 \\
\hline$\leq 4$ & Ref. & & Ref. & \\
\hline$>4$ & $1,05(0,83-1,31)$ & & $1,06(0,83-1,33)$ & \\
\hline Estado nutricional da mãe & & 0,500 & & 0,375 \\
\hline Eutrofia & Ref. & & Ref. & \\
\hline Desnutrição & $0,85(0,56-1,31)$ & & $0,83(0,54-1,28)$ & \\
\hline Excesso de peso & $1,08(0,87-1,35)$ & & $1,11(0,89-1,39)$ & \\
\hline \multicolumn{5}{|l|}{ Nível 4: variável de assistência social } \\
\hline Recebe Bolsa Família & & 0,894 & & 0,894 \\
\hline Não & Ref. & & Ref. & \\
\hline Sim & $1,01(0,81-1,27)$ & & $1,02(0,81-1,27)$ & \\
\hline
\end{tabular}

Ref.: referência.

Notas: foram avaliadas 1.185 crianças de 13 a 35 meses de idade. Nível 1: feito ajuste para as variáveis deste nível; Nível 2: feito ajuste para as variáveis deste nível e para as variáveis significantes $(p<0,05)$ do nível anterior (Nível 1); Nível 3: feito ajuste para as variáveis deste nível e para as variáveis significantes $(p<0,05)$ dos níveis anteriores (Níveis 1 e 2); Nível 4: feito ajuste para as variáveis deste nível e para as variáveis significantes $(p<0,05$ ) dos níveis anteriores (Níveis 1, 2 e 3). 


\section{Tabela 4}

Razões de prevalência (RP) brutas e ajustadas e intervalos de 95\% de confiança (IC95\%) para a associação entre características maternas e o padrão alimentar saudável em crianças da coorte de nascimento BRISA. São Luís, Maranhão, Brasil, 2010-2013.

\begin{tabular}{|c|c|c|c|c|}
\hline & RP bruta (IC95\%) & Valor de $p$ & RP ajustada (IC95\%) & Valor de $p$ \\
\hline \multicolumn{5}{|l|}{ Nível 1: variáveis da criança } \\
\hline Sexo & & 0,488 & & 0,479 \\
\hline Feminino & Ref. & & Ref. & \\
\hline Masculino & $0,92(0,75-1,14)$ & & $0,93(0,75-1,14)$ & \\
\hline Raça & & 0,375 & & 0,380 \\
\hline Não branca & Ref. & & Ref. & \\
\hline Branca & $1,11(0,88-1,40)$ & & $1,11(0,88-1,40)$ & \\
\hline \multicolumn{5}{|c|}{ Nível 2: variáveis socioeconômicas da mãe } \\
\hline Escolaridade (anos) & & $<0,001 *$ & & 0,009 * \\
\hline 1 a 4 & $0,13(0,29-0,62)$ & & $0,17(0,04-0,80)$ & \\
\hline 5 a 8 & $0,66(0,48-0,91)$ & & $0,75(0,53-1,06)$ & \\
\hline 9 a 11 & $0,63(0,50-0,81)$ & & $0,67(0,51-0,88)$ & \\
\hline 12 ou mais & Ref. & & Ref. & \\
\hline Classificação econômica brasileira & & 0,005 * & & 0,124 \\
\hline $\mathrm{A} / \mathrm{B}$ & Ref. & & Ref. & \\
\hline C & $0,87(0,67-1,13)$ & & $1,03(0,78-1,37)$ & \\
\hline $\mathrm{D} / \mathrm{E}$ & $0,57(0,40-0,81)$ & & $0,75(0,51-1,09)$ & \\
\hline \multirow{2}{*}{\multicolumn{5}{|c|}{$\begin{array}{l}\text { Nível 3: variáveis demográficas, reprodutivas e estado } \\
\text { nutricional da mãe }\end{array}$}} \\
\hline & & & & \\
\hline Situação conjugal & & 0,449 & & 0,346 \\
\hline Casada & Ref. & & Ref. & \\
\hline União consensual & $0,87(0,67-1,12)$ & & $1,06(0,80-1,39)$ & \\
\hline Sem companheiro & $0,98(0,71-1,34)$ & & $1,26(0,90-1,78)$ & \\
\hline Idade da mãe no parto (anos) & & 0,941 & & 0,725 \\
\hline$<20$ & $0,96(0,73-1,25)$ & & $1,11(0,81-1,51)$ & \\
\hline 20 a 34 & Ref. & & Ref. & \\
\hline$\geq 35$ & $1,02(0,70-1,48)$ & & $0,91(0,62-1,34)$ & \\
\hline Número de filhos & & 0,941 & & 0,332 \\
\hline 1 & $1,00(0,81-1,23)$ & & $0,86(0,68-1,09)$ & \\
\hline 2 a 4 & Ref. & & Ref. & \\
\hline$\geq 5$ & $0,88(0,43-1,79)$ & & $1,30(0,65-2,58)$ & \\
\hline Quantas pessoas moram na casa & & 0,451 & & 0,549 \\
\hline$\leq 4$ & Ref. & & Ref. & \\
\hline$>4$ & $0,91(0,73-1,15)$ & & $0,93(0,73-1,18)$ & \\
\hline Estado nutricional da mãe & & 0,817 & & 0,805 \\
\hline Eutrofia & Ref. & & Ref. & \\
\hline Desnutrição & $0,95(0,63-1,42)$ & & $1,07(0,71-1,60)$ & \\
\hline Excesso de peso & $1,06(0,85-1,31)$ & & $1,07(0,86-1,33)$ & \\
\hline \multicolumn{5}{|c|}{ Nível 4: variável de assistência social } \\
\hline Recebe Bolsa Família & & 0,096 & & 0,644 \\
\hline Não & Ref. & & Ref. & \\
\hline Sim & $0,82(0,64-1,04)$ & & $0,94(0,74-1,20)$ & \\
\hline
\end{tabular}

Ref.: referência.

Notas: foram avaliadas 1.185 crianças de 13 a 35 meses de idade. Nível 1: feito ajuste para as variáveis deste nível; Nível 2: feito ajuste para as variáveis deste nível e para as variáveis significantes $(p<0,05)$ do nível anterior (Nível 1); Nível 3: feito ajuste para as variáveis deste nível e para as variáveis significantes $(p<0,05)$ dos níveis anteriores (Níveis 1 e 2); Nível 4: feito ajuste para as variáveis deste nível e para as variáveis significantes $(p<0,05)$ dos níveis anteriores (Níveis 1, 2 e 3).

* Valor de $p<0,05$ 


\section{Tabela 5}

Razões de prevalência (RP) brutas e ajustadas e intervalos de 95\% de confiança (IC95\%) para a associação de características maternas com o padrão não saudável em crianças da coorte de nascimento BRISA. São Luís, Maranhão, Brasil, 2011-2013.

\begin{tabular}{|c|c|c|c|c|}
\hline & RP bruta (IC95\%) & Valor de $p$ & RP ajustada (IC95\%) & Valor de $p$ \\
\hline \multicolumn{5}{|l|}{ Nível 1: variáveis da criança } \\
\hline Sexo & & 0,952 & & 0,974 \\
\hline Feminino & Ref. & & Ref. & \\
\hline Masculino & $1,01(0,82-1,24)$ & & $1,00(0,82-1,23)$ & \\
\hline Raça & & 0,684 & & 0,684 \\
\hline Não branca & Ref. & & Ref. & \\
\hline Branca & $0,95(0,75-1,21)$ & & $0,95(0,75-1,21)$ & \\
\hline \multicolumn{5}{|c|}{ Nível 2: variáveis socioeconômicas da mãe } \\
\hline Escolaridade (anos) & & 0,383 & & 0,418 \\
\hline 1 a 4 & $1,28(0,71-2,33)$ & & $1,35(0,72-2,51)$ & \\
\hline 5 a 8 & $1,15(0,81-1,63)$ & & $1,25(0,85-1,85)$ & \\
\hline 9 a 11 & $0,95(0,70-2,33)$ & & $1,04(0,75-1,45)$ & \\
\hline 12 ou mais & Ref. & & Ref. & \\
\hline Classificação econômica brasileira & & 0,313 & & 0,386 \\
\hline $\mathrm{A} / \mathrm{B}$ & Ref. & & Ref. & \\
\hline C & $0,84(0,63-1,10)$ & & $0,79(0,58-10,7)$ & \\
\hline $\mathrm{D} / \mathrm{E}$ & $0,98(0,71-1,34)$ & & $0,87(0,61-1,24)$ & \\
\hline \multicolumn{5}{|c|}{$\begin{array}{l}\text { Nível 3: variáveis demográficas, reprodutivas e estado } \\
\text { nutricional da mãe }\end{array}$} \\
\hline Situação conjugal & & 0,456 & & 0,559 \\
\hline Casada & Ref. & & Ref. & \\
\hline União consensual & $1,10(0,84-1,44)$ & & $1,02(0,78-1,35)$ & \\
\hline Sem companheiro & $1,23(0,89-1,69)$ & & $1,17(0,84-1,63)$ & \\
\hline Idade da mãe no parto (anos) & & 0,008 * & & 0,005 * \\
\hline$<20$ & $1,04(0,82-1,34)$ & & $1,10(0,83-1,43)$ & \\
\hline 20 a 34 & Ref. & & Ref. & \\
\hline$\geq 35$ & $0,42(0,24-0,74)$ & & $0,40(0,23-0,70)$ & \\
\hline Número de filhos & & 0,560 & & 0,047 * \\
\hline 1 & $0,92(0,74-1,34)$ & & $0,80(0,64-1,00)$ & \\
\hline 2 a 4 & Ref. & & Ref. & \\
\hline$\geq 5$ & $1,18(0,68-2,06)$ & & $1,40(0,82-2,41)$ & \\
\hline Quantas pessoas moram na casa & & 0,392 & & 0,122 \\
\hline$\leq 4$ & Ref. & & Ref. & \\
\hline$>4$ & $0,90(0,72-1,14)$ & & $0,83(0,66-1,05)$ & \\
\hline Estado nutricional da mãe & & 0,074 & & 0,096 \\
\hline Eutrofia & Ref. & & Ref. & \\
\hline Desnutrição & $1,25(0,91-1,74)$ & & $1,25(0,91-1,72)$ & \\
\hline Excesso de peso & $0,85(0,68-1,07)$ & & $0,86(0,69-1,08)$ & \\
\hline \multicolumn{5}{|l|}{ Nível 4: variável de assistência social } \\
\hline Recebe Bolsa Família & & 0,241 & & 0,063 \\
\hline Não & Ref. & & Ref. & \\
\hline Sim & $0,87(0,69-1,10)$ & & $0,80(0,63-1,01)$ & \\
\hline
\end{tabular}

Ref.: referência.

Notas: foram avaliadas 1.185 crianças de 13 a 35 meses de idade. Nível 1: feito ajuste para as variáveis deste nível; Nível 2: feito ajuste para as variáveis deste nível e para as variáveis significantes $(p<0,05)$ do nível anterior (Nível 1); Nível 3: feito ajuste para as variáveis deste nível e para as variáveis significantes $(p<0,05)$ dos níveis anteriores (Níveis 1 e 2); Nível 4: feito ajuste para as variáveis deste nível e para as variáveis significantes ( $<$ 0,05) dos níveis anteriores (Níveis 1, 2 e 3).

* Valor de $p<0,05$. 
idade em municípios urbanos da Bahia. Um estudo de Gatica et al. 3 encontrou variância proporcional de $45,8 \%$, maior que a do presente trabalho.

O padrão alimentar comum brasileiro explicou o maior porcentual da variância proporcional. Esse padrão é formado por alimentos tradicionais da dieta do brasileiro, como também observado em outros estudos com crianças na primeira infância 3,21,22. Em um trabalho realizado em 1996 e 1999/2000 em municípios da Bahia, com crianças menores de 5 anos, o padrão tradicional foi denotado de "padrão 2" para a faixa etária de 12 a 23 meses e foi constituído por pão/biscoito, arroz, feijão, carnes e gordura, componentes presentes no padrão comum brasileiro deste estudo, com exceção das carnes 21. Gatica et al. 3, em um trabalho conduzido em 2005 e 2006 na cidade de Pelotas, Rio Grande do Sul, com crianças entre 12 e 24 meses, denominou de padrão "base" o padrão composto por arroz e feijão, por incluir alimentos básicos da alimentação dos brasileiros. A denominação "traditional" é habitualmente utilizada em pesquisas de outros países, mas os alimentos que compõem esta dieta variam de acordo com a cultura de cada país.

O segundo padrão identificado foi chamado de lácteo/mingau, integrado por leite de vaca e modificados, farináceos e açúcares; alimentos comumente utilizados na preparação de mingaus e espessantes. Nesse padrão, o leite materno apresentou carga fatorial negativa. A denominação desse padrão não foi encontrada na literatura pesquisada. Somente o estudo de Matos et al. ${ }^{21}$ caracterizou o seu padrão 1 para crianças entre 12 e 23 meses com os mesmos componentes do padrão lácteo/mingau do presente trabalho. Porém, não foi sugerida nomenclatura para esse padrão.

O padrão alimentar saudável é caracterizado pela presença de alimentos ricos em vitaminas, minerais, fibras e proteína, e baixo teor de açúcares e ácidos graxos trans. A denominação não foi semelhante à de outros padrões relatados na literatura brasileira para a faixa etária similar. Matos et al. 21 chamaram de "padrão 3" um padrão comparável ao saudável, composto por frutas, legumes/ tubérculos e macarrão. Gatica et al. 3 encontraram um terceiro padrão composto por carne, legumes/ verdura e batata/aipim, e este padrão foi chamado de "adulto" por incluir alimentos mais típicos da alimentação de crianças maiores e adultos. Outro estudo encontrou os componentes considerados saudáveis de forma isolada, a exemplo do padrão "vegetais" e "frutas" 22.

Algumas pesquisas internacionais com crianças da mesma faixa etária foram encontradas com padrão comparável ao saudável do presente estudo, dando destaque ao trabalho de Kristiansen et al. 23, cujo padrão foi bastante semelhante ao nosso. Nesse estudo norueguês, com crianças no segundo ano de vida, foi observado um tipo de padrão chamado de "saudável", que apresentou cargas fatoriais positivas para legumes, massas, arroz, peixes, aves, água e frutas 23 , havendo apenas a substituição das carnes e vísceras por peixes e aves, mas ambos são considerados alimentos proteicos. Em um estudo de coorte realizado na Inglaterra com crianças no segundo ano de vida, o padrão "saudável consciente" foi associado a alimentos frequentemente recomendados como base para uma dieta saudável, como frutas, legumes, ovos, nozes e sucos 24 .

O quarto padrão foi nomeado de não saudável. Esse padrão foi composto por refrigerantes/ sucos industrializados, biscoitos/bolos simples e lanches não saudáveis, alimentos ricos em açúcares simples, gorduras trans e aditivos químicos, não sendo o consumo destes nutrientes recomendado para esta faixa etária. A inclusão de refrigerantes/sucos artificiais nesse padrão é justificada por seu consumo crescente entre a população infantil 25. Um estudo baseado num recorte da Pesquisa Nacional de Demografia e Saúde (PNDS), realizado em 2006 com crianças de 2 a 5 anos, encontrou um percentual de consumo diário de refrigerantes ou sucos artificiais de 25,3\% 12. O consumo dessas bebidas açucaradas está associado ao aumento de peso, adiposidade e outras comorbidades, como o aumento da glicemia nas crianças 26,27 .

Os estudos brasileiros com crianças menores de 24 meses não extraíram um padrão que representasse somente alimentos considerados não saudáveis 3,21. Nos trabalhos de Matos et al. 21 e de Gatica et al. 3 com essa faixa etária, os únicos alimentos do padrão não saudável do presente estudo que participaram de algum dos padrões foram bolo e biscoitos simples. Do estudo de Gatica et al. 3 foi extraído o padrão "treats" em crianças de 48 meses, composto por salgadinhos, doces e chocolates. Um trabalho norueguês 23 com crianças de 24 meses identificou um padrão "não saudável" composto por pizza, hambúrguer, batata frita, doces, sobremesas, bolos e biscoito. Tais dados apontam para a introdução precoce desses alimentos considerados prejudiciais para a saúde na dieta infantil. 
Diferenças nos alimentos que compõem os padrões com a mesma denominação encontrados na literatura podem ser explicadas pela subjetividade inerente aos métodos de análise de componentes principais.

Neste estudo, a maioria dos componentes encontrados reflete a percepção subjetiva dos principais grupos de alimentos da infância, coincidindo com as práticas mais comuns de alimentação infantil baseadas em leites, mingaus e espessantes, sopas, lanches à base de pão, biscoitos, frutas, sucos e a preferência por arroz e feijão como base alimentar. O "não saudável” destaca-se como o padrão menos comum e menos adequado para essa faixa etária.

A carga fatorial do leite materno no padrão lácteo/mingau foi negativa, indicando a substituição do leite materno por leites e/ou mingaus. Essa tendência acentuada na redução do consumo de leite materno pode ser observada quando esses grupos se expressam nos padrões alimentares extraídos para essa faixa etária 21,28. Num trabalho realizado no Nordeste, o leite materno compôs o primeiro padrão de consumo das crianças de 6 a 24 meses, sempre com carga fatorial negativa, e deixou de compor qualquer padrão de consumo a partir dos 24 meses de idade 21 . No Reino Unido, onde foram estudados os padrões alimentares de crianças aos 6 e 12 meses de idade, o leite materno apareceu com escore negativo aos 6 meses e, aos 12 meses de idade o leite materno não apareceu em nenhum dos componentes 28 . Essa constatação reafirma os achados recentes de regimes alimentares que ainda se contrapõem às recomendações de manutenção do consumo de leite materno pelo menos até os dois anos ou mais 29 .

Para o padrão comum brasileiro, foi encontrada associação para as variáveis maternas idade, escolaridade e número de filhos. Mães com mais de 5 filhos apresentaram maior aderência a esse padrão. Tal achado pode ser explicado pelo fato desse padrão ser composto por alimentos de baixo custo e ser de fácil acesso e preparação, o que facilita sua oferta para um maior número de pessoas. Filhos de mães de menor escolaridade e de adolescentes apresentaram maior aderência a esse padrão. O padrão comum brasileiro, apesar de contar com componentes importantes da alimentação (como o arroz e o feijão), apresenta pouca variedade de nutrientes, sendo rico basicamente em amido (pães, arroz e biscoitos) e gorduras (margarina e manteiga), além de não incluir alimentos saudáveis, como frutas e hortaliças 4 . Dessa forma, essa associação entre menor idade materna e maior aderência a esse padrão pode ser explicada, possivelmente, pelo fato de mães mais velhas apresentarem melhor discernimento, interesse e maior acesso às informações sobre a alimentação de seu filho em relação às mais jovens.

Não foi encontrado estudo com associação entre as variáveis paridade e idade da mãe com o padrão comum brasileiro ou com padrões que apresentem componentes similares. Em relação à escolaridade, Nobre et al. 14 também encontraram maior frequência de consumo de alimentos do padrão "dieta mista”, similar ao padrão tradicional, entre filhos de mães com menor escolaridade, estudando crianças de 4 a 11 anos.

Nenhuma das características maternas investigadas mostrou associação com o padrão lácteo/ mingau. Esse fato indica que a oferta de alimentos desse padrão independe das características maternas, sendo que esse padrão reflete monotonia alimentar e a substituição de alimentos importantes para a saúde da criança por lácteos e mingaus.

A aderência ao padrão saudável variou de acordo com a escolaridade da mãe, com maior oferta dos alimentos deste padrão a filhos de mães com maior escolaridade. Maior acesso a informações e conhecimentos sobre a importância do consumo de alimentos saudáveis na infância em mães com maior escolaridade pode explicar tal achado. Além disso, essas mães são menos vulneráveis à publicidade de alimentos não saudáveis.

Já é sabido que hábitos alimentares mais saudáveis em pré-escolares estão associados à escolaridade materna. Gatica et al. 3 encontraram maior consumo do padrão “carne e vegetais", alimentos do padrão saudável no presente estudo, em filhos de mães com 8 anos ou mais de escolaridade. Lioret et al. 30, estudando crianças de até 24 meses, também encontraram maior aderência do padrão considerado saudável em filhos de mulheres com maior escolaridade.

Para o padrão não saudável, foi registrada associação para a idade materna e paridade, com os filhos únicos e de mães com mais de 35 anos tendo menor aderência a este padrão. Mães com somente um filho têm maior tempo, dedicação e recursos para planejar com maior qualidade a alimentação do seu filho, reduzindo assim a necessidade de compra de alimentos prontos e fast food. Além disso, o consumo alimentar do filho mais velho pode influenciar na introdução alimentar precoce de alimen- 
tos industrializados e guloseimas para a criança mais nova. Um estudo norueguês com crianças de 2 anos de idade encontrou maior aderência ao padrão "não saudável" em filhos de mães multíparas, resultado similar ao do presente trabalho 23 .

Menor aderência ao padrão "não saudável" associado com maior idade materna pode ser justificada porque possivelmente mães mais velhas apresentem um melhor discernimento, interesse e maior acesso às informações sobre a alimentação de seu filho, ao contrário daquelas mais jovens. A diferença do hábito alimentar entre as mães mais velhas e mais jovens também justifica tal achado, pois mães mais jovens têm maior acesso e preferência a alimentos industrializados e guloseimas, o que pode refletir na alimentação do seu filho. Kristiansen et al. 23 encontraram resultado similar, pois filhos de mães maiores de 24 anos apresentaram menor aderência ao padrão “unhealthy", padrão este composto por fast food, guloseimas e bebidas açucaradas. No estudo de Gatica et al. 3, padrão comparável ao "não saudável” foi observado somente para crianças com 48 meses de idade, sendo também encontrada menor aderência em filhos de mães mais velhas, maiores de 30 anos.

Quatro padrões alimentares foram identificados entre as crianças estudadas, sendo que três destes padrões representam alimentos ricos em amido (pães, farináceos, biscoitos e bolos simples), gordura saturada (margarina, manteiga, leite de vaca integral e lanches não saudáveis) e açúcar simples (açúcar/mel, refrigerantes/sucos artificiais e lanches não saudáveis) e pobres em nutrientes importantes para o bom desenvolvimento das crianças, como vitaminas, minerais e fibras. Chamou a atenção o aparecimento do leite materno com carga negativa no padrão lácteo/mingau, apontando para a sua substituição pelos leites de vaca e modificados. Multiparidade, menor escolaridade materna e idade materna menor de 20 anos foram associadas ao menor consumo de alimentos considerados saudáveis e importantes para o desenvolvimento infantil.

Esses resultados apontam para a necessidade de estímulo à melhoria da alimentação das crianças, que requer políticas públicas de incentivo ao maior consumo de alimentos saudáveis, à manutenção do aleitamento materno até os dois anos de idade ou mais e às atividades de educação nutricional voltadas para as mães de crianças na primeira infância. Além disso, políticas intersetoriais, como maior acesso à educação e planejamento familiar, devem ser prioritárias por refletirem em diversas áreas, inclusive na qualidade da alimentação das crianças.

\section{Colaboradores}

E. G. Bogea, S. P. M. Arruda e A. A. M. Silva participaram da concepção e projeto, análise e interpretação dos dados, redação e revisão crítica relevante do conteúdo intelectual do artigo, aprovação final da versão a ser publicada. M. L. B. Martins, W. R. C. Carvalho e A. K. T. C. França colaboram na redação e revisão crítica relevante do conteúdo intelectual do artigo e aprovação final da versão a ser publicada.

\section{Agradecimentos}

Agradecimentos às Fundações de Amparo à Pesquisa do Maranhão (FAPEMA) e de São Paulo (FAPESP), pelo patrocínio e viabilização da pesquisa. Ao Conselho Nacional de Desenvolvimento Científico e Tecnológico (CNPq), pelo financiamento. Agradecimentos às crianças e mães que compõem o estudo de coorte BRISA.

\section{Informações adicionais}

ORCID: Eduarda Gomes Bogea (0000-0003-26568238); Maylla Luanna Barbosa Martins (0000-00026402-3899); Wyllyane Rayana Chaves Carvalho (0000-0001-7704-9951); Soraia Pinheiro Machado Arruda (0000-0002-3918-4738); Ana Karina Teixeira da Cunha França (0000-0002-4460-2631); Antonio Augusto Moura da Silva (0000-0003-49685138). 


\section{Referências}

1. Baldissera R, Issler RMS, Giugliani ERJ. Efetividade da Estratégia Nacional para Alimentação Complementar Saudável na melhoria da alimentação complementar de lactentes em um município do Sul do Brasil. Cad Saúde Pública 2016; 32:e00101315.

2. Jaime PC, Frias PG, Monteiro HOC, Almeida PVB, Malta DC. Healthcare and unhealthy eating among children aged under two years: data from the National Health Survey, Brazil, 2013. Rev Bras Saúde Matern Infant 2016; 16:14957.

3. Gatica G, Barros AJ, Madruga S, Matijasevich A, Santos IS. Food intake profiles of children aged 12, 24 and 48 months from the 2004 Pelotas (Brazil) birth cohort: an exploratory analysis using principal components. Int $\mathrm{J} \mathrm{Be}$ hav Nutr Phys Act 2012; 9:43.

4. D'Innocenzo S, Marchioni DML, Prado MS Matos SMA, Pereira SRS, Barros AP, et al. Condições socioeconômicas e padrões alimentares de crianças de 4 a 11 anos: estudo SCAALA - Salvador/Bahia. Rev Bras Saúde Matern Infant 2011; 11:41-9.

5. Fisberg M, Del'Arco APWT, Previdelli A, Tosatti AM, Nogueira-de-Almeida CA. Hábito alimentar nos lanches intermediários de crianças pré-escolares brasileiras: estudo em amostra nacional representativa. International Journal of Nutrology 2015; 8:58-71.

6. Vaz-Tostes MG, Pires PCC, Paula AH, Barros AA, Freitas FV, Santana HMP, et al. Estado nutricional relativo ao ferro, zinco e vitamina A de pré-escolares inseridos em um programa de educação alimentar e nutricional. HU Rev 2015; 41:163-70.

7. Borges CA, Slater B, Santaliestra-Pasías AM, Mouratidou T, Huybrechts I, Widhalm K, et al. Dietary patterns in European and Brazilian adolescents: comparisons and associations with socioeconomic factors. Nutrients 2018; 10:57.

8. Carvalho CA, Fonsêca PCA, Nobre LN, Priore SE, Franceschini SCC. Metodologias de identificação de padrões alimentares a posteriori em crianças brasileiras: revisão sistemática. Ciênc Saúde Colet 2016; 21:143-54.

9. Olinto MTA. Padrões alimentares: análise de componentes principais. In: Kac G, Sichieri $\mathrm{R}$, Gigante DP, organizadores. Epidemiologia nutricional. Rio de Janeiro: Editora Fiocruz/ Editora Atheneu; 2007. p. 213-25.

10. Arruda SP, Silva AA, Kac G, Goldani M, Bettiol H, Barbieri M. Socioeconomic and demographic factors are associated with dietary patterns in a cohort of young Brazilian adults. BMC Public Health 2014; 14:654.

11. McPhie S, Skouteris H, Daniels L, Jansen E. Maternal correlates of maternal child feeding practices: a systematic review. Matern Child Nutr 2012; 10:18-43.
12. Alves MN, Muniz LC, Vieira MFA. Consumo alimentar entre crianças brasileiras de dois a cinco anos de idade: Pesquisa Nacional de Demografia e Saúde (PNDS), 2006. Ciênc Saúde Colet 2013; 18:3369-77.

13. Okubo H, Miyake Y, Sasaki S, Tanaka K, Murakami K, Hirota Y. Dietary patterns in infancy and their associations with maternal socioeconomic and lifestyle factors among 758 Japanese mother-child pairs: the Osaka Maternal and Child Health Study. Matern Child Nutr 2014; 10:213-25.

14. Nobre LN, Lamounier JA, Franceschini SCC. Padrão alimentar de pré-escolares e fatores associados. J Pediatr (Rio J.) 2012; 88:129-36.

15. Silva AA, Batista RF, Simões VM, Thomaz EBAF, Ribeiro CCC, Lamy Filho $F$, et al Changes in perinatal health in two birth cohorts (1997/1998 and 2010) in São Luís, Maranhão State, Brazil. Cad Saúde Pública 2015; 31:1437-50.

16. Holanda LB, Barros Filho AA. Métodos aplicados em inquéritos alimentares. Rev Paul Pediatr 2006; 24:62-70.

17. Pinheiro ABV, Lacerda EMA, Benzecry EH, Gomes MCS, Costa VM. Tabela para avaliação de consumo alimentar em medidas caseiras. Rio de Janeiro: Editora Atheneu; 2004.

18. Drewett RF, Woolridge MW, Jackson DA, Imong SM, Mangklabruks A, Wongsawasdii L, et al. Relationships between nursing patterns, supplementary food intake and breast-milk intake in a rural Thai population. Early Hum Dev 1989; 20:13-23.

19. Instituto Brasileiro de Geografia e Estatística. Pesquisa de Orçamentos Familiares 20082009: tabela de medidas referidas para os alimentos consumidos no Brasil. Rio de Janeiro: Instituto Brasileiro de Geografia e Estatística; 2011.

20. Seaman SR, White IR. Review of inverse probability weighting for dealing with missing data. Stat Methods Med Res 2013; 22:278-95.

21. Matos SMA, Barreto ML, Rodrigues LC, Oliveira VA, Oliveira LPM, D'Innocenzo S, et al. Padrões alimentares de crianças menores de cinco anos de idade residentes na capital e em municípios da Bahia, Brasil, 1996 e 1999/2000. Cad Saúde Pública 2014; 30:44-54.

22. Souza RLV, Madruga SW, Gigante DP, Santos IS, Barros AJD, Assunção MCF. Padrões alimentares e fatores associados entre crianças de um a seis anos de um município do Sul do Brasil. Cad Saúde Pública 2013; 29:2416-26.

23. Kristiansen AL, Lande B, Sexton JA, Andersen LF. Dietary patterns among Norwegian 2-year-olds in 1999 and in 2007 and associations with child and parent characteristics. $\mathrm{Br} \mathrm{J}$ Nutr 2013; 110:135-44. 
24. Northstone K, Emmett P. The associations between feeding difficulties and behaviours and dietary patterns at 2 years of age: the ALSPAC cohort. Matern Child Nutr 2013; 9:533-42.

25. Instituto Brasileiro de Geografia e Estatística. Pesquisa de Orçamentos Familiares 20082009: análise do consumo alimentar pessoal no Brasil. Rio de Janeiro: Instituto Brasileiro de Geografia e Estatística; 2011.

26. Gibson S. Sugar-sweetened soft drinks and obesity: a systematic review of the evidence from observational studies and interventions. Nutr Res Rev 2008; 21:134-47.

27. Vartanian LR, Schwartz MB, Brownell KD. Effects of soft drink consumption on nutrition and health: a systematic review and meta-analysis. Am J Public Health 2007; 97:667-75.
28. Robinson S, Marriott L, Poole J, Crozier S, Borland S, Lawrence W, et al. Dietary patterns in infancy: the importance of maternal and family influences on feeding practice. $\mathrm{Br} \mathrm{J}$ Nutr 2007; 98:1029-37.

29. Palmeira PA, Santos SMC, Vianna RPT. Prática alimentar entre crianças menores de dois anos de idade residentes em municípios do semiárido do Estado da Paraíba, Brasil. Rev Nutr 2011; 24:553-63.

30. Lioret S, Betoko A, Forhan A, Charles MA, Heude B, de Lauzon-Guillain B. Dietary patterns track from infancy to preschool age: cross-sectional and longitudinal perspectives. J Nutr 2015; 145:775-82. 


\section{Abstract}

This study sought to identify eating patterns among children and to verify their association with maternal characteristics. We studied 1,185 children aged 13 to 35 months. Food consumption was investigated using the $24 \mathrm{~h}$ dietary recall and the eating patterns were identified using principal components factor analysis, followed by orthogonal varimax rotation. We carried out a hierarchical modeling using poisson regression with robust variance estimates in order to estimate prevalence ratios. We identified four eating patterns: common Brazilian, dairy/porridge, healthy and unhealthy. The variance explained by these factors was of 34.3\% and the common Brazilian pattern was the one that most contributed to proportional variance. We highlight the dairy/porridge pattern, in which breast milk had negative factorial loading, suggesting its substitution by cow and modified milk. The data reveal that the characterization of children's eating patterns diverges according to maternal characteristics, such as age, educational level and number of children. Three of the four patterns we found are represented by foods rich in starch, saturated fat and simple sugar and poor in vitamins, minerals and fibers. Multiparity, lower educational levels and maternal age under 20 years were associated with a lower consumption of foods considered to be healthy and important in child development.

Child Nutrition; Food Consumption; Mothers; Statistical Factor Analysis

\section{Resumen}

El objetivo fue identificar los estándares alimentarios en niños y verificar su asociación con características maternas. Se estudiaron a 1.185 niños de 13 a 35 meses de edad. El consumo alimentario se investigó mediante un recordatorio alimentario de 24 horas, $y$ los estándares alimentarios se identificaron mediante un análisis factorial por componentes principales, seguidos de una rotación ortogonal varimax. Se utilizó un modelado jerarquizado mediante la regresión de Poisson con estimativa robusta de la variancia para estimar razones de prevalencias. Se identificaron cuatro estándares alimentarios: brasileño común, lácteo/papillas, saludable y no saludable. La variancia explicada por esos factores fue 34,3\%, donde el estándar común brasileño fue el que más contribuyó a la variancia proporcional. Se destaca el estándar lácteo/papilla, donde la leche materna presentó una carga factorial negativa, apuntando su sustitución por leches de vaca y modificadas. Los datos revelan que la caracterización de los estándares alimentarios de los niños diverge según las características maternas, como la edad, escolaridad y número de hijos. Tres de los cuatro estándares encontrados se representan por alimentos ricos en almidón, grasa saturada y azúcar común y pobre en vitaminas, minerales y fibras. Multiparidad, menor escolaridad materna y edad materna inferior a 20 años se asociaron a un menor consumo de alimentos considerados saludables e importantes para el desarrollo infantil.

Nutrición del Niño; Consumo de Alimentos; Madres; Análisis Factorial
Recebido em 16/Abr/2018

Versão final reapresentada em 01/Nov/2018 Aprovado em 21/Nov/2018 